\title{
ABOUT THE EXISTENCE OF MILNOR FIBRATIONS
}

\author{
J. L. CISNEROS-MOLINA AND R. N. ARAÚJO DOS SANTOS
}

\begin{abstract}
The aim of the present article is to review the results about the existence of Milnor fibrations for complex and real singularities and some generalizations in several settings that have been developed recently. After recalling the classical theorems by Milnor, we start with the complex case for germs of functions and maps, then we continue with the real analytic cases and list some open questions.
\end{abstract}

\section{INTRODUCTION}

In his now classical book [Mi1] Milnor proves a Fibration Theorem, which associates a locally trivial fibration to each singular point of a complex hypersurface. It is a very useful and fundamental tool to understand its local topological behavior.

Given a complex holomorphic function

$$
f:\left(\mathbb{C}^{n}, 0\right) \rightarrow(\mathbb{C}, 0),
$$

let $V(f)=f^{-1}(0)$, denote by $\sum(f)$ the critical locus of $f$ and suppose $0 \in \sum(f)$. Let $S_{\epsilon}^{2 n-1}$ be the sphere centered at $0 \in \mathbb{C}^{n}$ of radius $\epsilon>0$. The set $K_{\epsilon}:=V(f) \cap S_{\epsilon}^{2 n-1}$ is called the link of the singularity at the origin.

Theorem 1.1 (Milnor Fibration Theorem [Mi1]). There exists a small $\epsilon_{0}>0$, such that, for all $0<\epsilon \leq \epsilon_{0}$, the map

$$
\frac{f}{|f|}: S_{\epsilon}^{2 n-1} \backslash K_{\epsilon} \rightarrow S^{1},
$$

is the projection of a smooth locally trivial fibration. Furthermore, if $0 \in \mathbb{C}^{n}$ is an isolated critical point of $f$, then the fibers of this fibrations have the homotopy type of a bouquet of spheres of dimension $n-1$ and the topological closure of each fiber is the link $K_{\epsilon}$.

2000 Mathematics Subject Classification. 32S55, 32S60, 58K15.

Key words and phrases. Milnor fibration, topology of singularities, stratifications.

The first author is a Regular Associate of the International Centre for Theoretical Physics, Trieste, Italy. 
Let us see one example in the case $n=2$.

Example 1.2. Consider the holomorphic map $f:\left(\mathbb{C}^{2}, 0\right) \rightarrow(\mathbb{C}, 0)$, given by $f(z, w)=z^{2}+w^{3}$. Since $\nabla f(z, w)=(0,0)$ implies $(z, w)=$ $(0,0)$, then we have that $\sum(f)=\{(0,0)\}$, i. e., $f$ has an isolated critical point at the origin. Since the function $f$ is weighted-homogeneous of type $(3,2 ; 6)$, it means that $V(f) \backslash\{(0,0)\}$ is an analytic manifold that cuts transversally all spheres of any radius. Without loss of generality, we can choose the sphere $S_{\sqrt{2}}^{3}=\left\{(z, w) \in \mathbb{C}^{2}:\|z\|^{2}+\|w\|^{2}=2\right\}$.

Using the transversality theorem, the link $K_{\sqrt{2}}=V(f) \cap S_{\sqrt{2}}^{3}$ is a real analytic submanifold in $S_{\sqrt{2}}^{3}$ of dimension one, i.e, a regular curve.

Let us describe this link.

$$
\text { If }(z, w) \in K_{\sqrt{2}} \Rightarrow\left\{\begin{array}{l}
|z|^{2}=|w|^{3}, \\
|z|^{2}+|w|^{2}=2 .
\end{array}\right.
$$

This system provides the equation $X^{3}+X^{2}-2=0$ on the variable $X=|w|$, which can be factorized as $X^{3}+X^{2}-2=(X-1)\left(X^{2}+2 X+2\right)$. Hence this equation has only one real solution $X=|w|=1$ and two others which are complex conjugated.

Considering the real solution $|w|=1$ and using the second equation $|z|^{2}+|w|^{2}=2$, we get $|z|=1$. It means that, the link $K_{\sqrt{2}}$ lives on the torus $S^{1} \times S^{1}=\left\{\left(e^{i \theta}, e^{i \phi}\right) \in S_{\sqrt{2}}^{3}:(\theta, \phi) \in[0,2 \pi] \times[0,2 \pi]\right\}$.

Now consider the parametrization $\varphi:[0,12 \pi] \rightarrow V(f) \cap S_{\sqrt{2}}^{3}$ given by $\varphi(t)=\left(e^{\frac{i t}{2}}, e^{\frac{i t}{3}}\right)$ (see the Figure 1 below). We can see clearly that the intersection is the well-known $(2,3)$-torus knot also called the "trefoil knot".

According to Milnor's theorem this curve is the topological closure or the boundary of the fibers.

This example is a particular case of weighted-homogeneous polynomials in $n$ complex variables called Brieskorn-Pham polynomials

$$
z_{1}^{a_{1}}+\cdots+z_{n}^{a_{n}}, \quad n>0, a_{j} \in \mathbb{N}, a_{j} \geq 2, j=1, \ldots, n .
$$
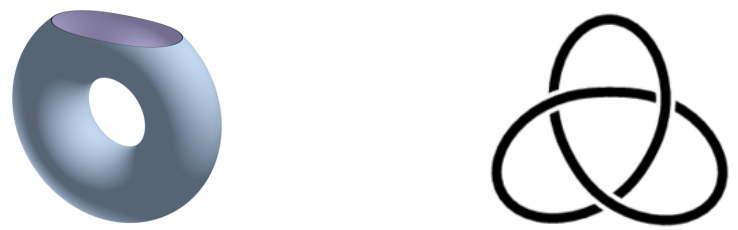

Figure 1. Fibre and link of $V\left(z^{2}+w^{3}\right)$. 
1.1. Milnor fibration for real singularities. Milnor wrote an article [Mi] showing that for a real polynomial map germ

$$
f:\left(\mathbb{R}^{n}, 0\right) \rightarrow\left(\mathbb{R}^{k}, 0\right), \quad n \geq k \geq 2 .
$$

with $0 \in \mathbb{R}^{n}$ an isolated critical point, it is always possible to associate a locally trivial fibration. This article was never published but its results appeared in $\S 11$ of [Mi1].

In what follow we will describe this fibration, for this, denote by $B_{\epsilon}$ the real closed ball of dimension $n$ centered at $0 \in \mathbb{R}^{n}$ and radius $\epsilon$, let $S_{\eta}^{k-1}$ be the sphere centered at $0 \in \mathbb{R}^{k}$ of radius $\eta>0$. Then Milnor's result is:

Theorem 1.3 ([Mi, Mi1] $)$. There exists a small $\epsilon_{0}>0$, such that for all $0<\epsilon \leq \epsilon_{0}$, there exists $\eta$, with $0<\eta \ll \epsilon$, such that the map

$$
\frac{1}{\eta} f \mid: B_{\epsilon} \cap f^{-1}\left(S_{\eta}^{k-1}\right) \rightarrow S^{k-1}
$$

is the projection of a smooth locally trivial fibration.

Let $S_{\epsilon}^{n-1}=\partial B_{\epsilon}$ and as before, let $V(f)=f^{-1}(0)$ and define the link by $K_{\epsilon}:=V(f) \cap S_{\epsilon}^{n-1}$. Since $0 \in \mathbb{R}^{n}$ is an isolated singularity of $f$, then $0 \in \mathbb{R}^{k}$ is a regular value of $f \mid: S_{\epsilon}^{n-1} \rightarrow \mathbb{R}^{k}$, if $\epsilon>0$ is small enough. Let $N\left(K_{\epsilon}\right)$ stand for an open tubular neighborhood of $K_{\epsilon}$ in the sphere. Milnor used the flow of an appropriated vector field in $B_{\epsilon} \backslash V(f)$, to construct a diffeomorphism from $B_{\epsilon} \cap f^{-1}\left(S_{\eta}^{k-1}\right)$ to $S_{\epsilon}^{n-1} \backslash N\left(K_{\epsilon}\right)$, getting in this way, up to diffeomorphism, a fibration $S_{\epsilon}^{n-1} \backslash N\left(K_{\epsilon}\right) \rightarrow S^{k-1}$. It is not difficult to see that one can always extend this fibration into the open tubular neighborhood $N\left(K_{\epsilon}\right)$ to get a fibration

$$
S_{\epsilon}^{n-1} \backslash K_{\epsilon} \rightarrow S^{k-1}
$$

Even in his book [Mi1, p. 100], Milnor comments that the major weakness of Theorem 1.3 is that the hypothesis is so strong that is very difficult to find examples, except those that comes from holomorphic functions and posed the problem to find dimensions $n \geq k \geq 2$ for which there exists non-trivial examples.

This was solved for $k=2$ in [Lo] for $n$ even and in [CL] for $n$ odd, also in [CL] was determined all the possible pairs $(n, k)$ for which there exist real map germs satisfying Milnor's hypothesis, but no explicit examples of such singularities were given. The first explicit non-trivial example for the case $k=2$ was given in [A]. 
On the other hand, some natural questions come along the construction of fibration (2). In order to propose one of them, we will do below some considerations.

It follows from the construction of fibration (2) that one completely loses control over the projection map; i.e. Theorem 1.3 only ensures the existence of fibration (2) for some projection map and unlike the complex case it is completely unknown.

In other words, on real settings, there is not any a priori reason to expect that this projection must be the canonical map $\frac{f}{|f|}$, as in the complex case. In fact, Milnor gave an example (see [Mi1, p. 99]) showing that, in general, this map fails to be the projection map of fibration (2). This motivated the following definition.

Definition 1.4 ([RSV], [S2]). Let $n \geq 2$. Given a real analytic map germ $f:\left(\mathbb{R}^{n}, 0\right) \rightarrow\left(\mathbb{R}^{2}, 0\right)$, with isolated critical point at the origin, we say that $f$ satisfies the strong Milnor condition at 0 , if and only if, for all $\epsilon>0$ sufficiently small the map

$$
\frac{f}{\|f\|}: S_{\epsilon}^{n-1} \backslash K_{\epsilon} \rightarrow S^{1}
$$

is the projection of a locally trivial fibration.

So, a natural question is: Are there real analytic maps which satisfy the strong Milnor condition?

By Theorem 1.1 we have a partial positive answer: Just look at any holomorphic function $f:\left(\mathbb{C}^{n}, 0\right) \rightarrow(\mathbb{C}, 0)$, with isolated singularity at the origin, as a pair of real analytic maps $f=(\Re(f), \Im(f))$, where $\Re(f)$ and $\Im(f)$ are the real and imaginary part of $f$, respectively.

What about real analytic maps which do not come from holomorphic ones?

This introduces the problem of study natural conditions under which a real analytic map germ $f=\left(f_{1}, f_{2}\right):\left(\mathbb{R}^{n}, 0\right) \rightarrow\left(\mathbb{R}^{2}, 0\right), n \geq 2$, satisfies the strong Milnor condition, i.e,

How big the class of real analytic maps which satisfy the strong Milnor condition is?

As far as we know, this problem was first approached in [Ja, Ja1] by A. Jacquemard. There the author proposed two sufficient conditions, 
one geometric and one algebraic, that imply the strong Milnor condition. Some years later, Seade in [S] and Seade, Ruas and Verjovsky in [RSV], used a pencil of real analytic hypersurfaces canonically associated to the corresponding map germs to study singularities with the strong Milnor condition (see §2.2). Even in [RSV], the authors provided a method to find an infinite family of singularities satisfying this condition, in particular, the twisted Brieskorn-Pham polynomials, which are real analytic analogues to Brieskorn-Pham polynomials (1) (see Example 3.8).

In $[\mathrm{RS}]$, using different tools of singularity theory and stratification theory the authors refined the previous argument and proved that under a weaker condition (Bekka's (c)-regularity) on a stratification of an analytic set given by projections of the map $f$, also is possible to guarantee the existence of this condition. See [RS] for details. Afterwards, in [Sa] the author got a slight improvement of the last one using only the called (m)-condition. Actually, it was inspired from K. Bekka and Koike's result proving that (c)-regularity imply (m)-condition.

Recently, in [CSS, ST, CSS1] necessary and sufficient conditions were given to assure that given a real analytic map $f:\left(\mathbb{R}^{n}, 0\right) \rightarrow\left(\mathbb{R}^{k}, 0\right)$, with $n \geq k \geq 2$, the map $\frac{f}{\|f\|}: S_{\epsilon}^{n-1} \backslash K_{\epsilon} \rightarrow S^{p-1}$ is the projection of a locally trivial fibration. We will explain these results in more details on $\S 3.4$ and $\S 3.5$.

In what follows, we intend to present some interesting generalizations, in the authors point of view, of Milnor fibrations on complex and real cases, which have been developed in these last years. We will start with the complex case for germs of functions and maps and after that we will move to the real analytic cases and list some open questions. As a complementary reading we recommend the interesting survey article [S3].

\section{Milnor FiBRAtions FOR COMPleX FUnCTIONS AND MAPS}

In this section we will be concerned with the complex case.

2.1. Milnor Fibration on complex analytic sets. Let us start with an improvement of Milnor Fibration Theorem [Mi1] for complex functions done by Lê Dũng Tráng in [Le1], which generalizes the existence of Milnor fibrations on complex analytic sets.

Let $X$ be an analytic subset of an open neighbourhood $U$ of the origin 0 in $\mathbb{C}^{n}$. Let $f:(X, 0) \rightarrow(\mathbb{C}, 0)$ be holomorphic and set $V=f^{-1}(0)$. Let $B_{\epsilon}$ be a ball in $U$ of sufficiently small radius $\epsilon>0$, centred at $0 \in \mathbb{C}^{n}$ and $D_{\eta}-\{0\}$ be the punctured disc of radius $\eta$ in $\mathbb{C}$. 
Theorem 2.1 (Milnor-Lê Fibration [Le1]). For all $\epsilon>0$ small enough, there exists $\eta$, with $0<\eta \leq \epsilon$, such that

$$
f \mid: B_{\epsilon} \cap X \cap f^{-1}\left(D_{\eta}-\{0\}\right) \rightarrow D_{\eta}-\{0\}
$$

is a topological locally trivial fibration.

Idea of proof. Let $\mathcal{S}$ be a Whitney stratification of $X$, choose $\epsilon>0$ small enough such that the closed ball $B_{\epsilon}$ intersects only a finite number of strata of $X$ and such that the sphere $S_{\epsilon}$, boundary of $B_{\epsilon}$, intersects such strata transversally. Moreover, according to [Hi], we can always choose this stratification in such way that $V$ is union of strata and satisfies Thom's $A_{f}$-condition. This implies that for $0<\eta \ll \epsilon$ the fibres of the map

$$
f \mid: B_{\epsilon} \cap X \cap f^{-1}\left(D_{\eta}-\{0\}\right) \rightarrow D_{\eta}-\{0\},
$$

intersect transversally the strata of $X \cap S_{\epsilon}$ and that it is a stratified submersion. Now the result follow from Thom-Mather First Isotopy Theorem [Mat].

Lê also observed that $\mathrm{H}$. Hamm in $[\mathrm{H}]$ proved that this topological fibration becomes a smooth fibration, if $X \backslash V$ is a non-singular analytic set in $\mathbb{C}^{N}$.

As stated below, this result was generalized by E. Looijenga in [Lo1] for complex analytic maps germs $f:\left(\mathbb{C}^{n}, 0\right) \rightarrow\left(\mathbb{C}^{p}, 0\right)$ that locally define an Isolated Complete Intersection Singularity (ICIS). This means that in a sufficient small ball $B_{\epsilon} \subset \mathbb{C}^{n}$ the intersection $\left(f^{-1}(0)-\{0\}\right) \cap$ $B_{\epsilon}$ is an analytic manifold. Set $C(f)=f\left(\sum(f)\right)$ be the discriminant set of $f$.

Theorem 2.2 ([Lo1]). There exists $\epsilon_{0}$, such that for all $0<\epsilon \leq \epsilon_{0}$, there exists $0<\eta \ll \epsilon$, such that the projection map

$$
f \mid: B_{\epsilon} \cap f^{-1}\left(D_{\eta}-C(f)\right) \rightarrow D_{\eta}-C(f)
$$

is a smooth locally trivial fibration.

One has the following natural

Question 1. What are the good conditions under which it is possible to guarantee the existence of Milnor fibration for a complete intersection with non-isolated singularity?

More recently in [Ga], using the tool of integral closure of modules, T. Gaffney did a beautiful and interesting approach to this question. 
There is also a generalization of Theorem 1.1 on complex analytic sets, which is implicit in the work of Lê Dũng Tráng [Le] and a weaker form of it is also given in [Du, Thm. 3.9].

Let $L_{X \epsilon}=X \cap S_{\epsilon}$ be the link of $X$ and let $L_{f_{\epsilon}}=X \cap V \cap S_{\epsilon}$ be the link of $f$ in $X$.

Theorem 2.3. There exists $\epsilon_{0}>0$, such that, for all $0<\epsilon \leq \epsilon_{0}$, the map

$$
\phi=\frac{f}{|f|}: L_{X \epsilon} \backslash L_{f_{\epsilon}} \longrightarrow S^{1} .
$$

is a locally trivial fibration.

A proof of this theorem and the fact that the fibration (4) is equivalent to the restriction $f \mid: B_{\epsilon} \cap X \cap f^{-1}\left(\partial D_{\eta}\right) \rightarrow \partial D_{\eta}$ of fibration (3) can be found in [CSS] (see Theorem 2.5 below).

2.2. Refinements of Milnor Fibration Theorems. In [CSS] the authors give some refinements of Theorems 2.1 and 2.3.

As in $\S 2.1$, let $X$ be an analytic subset of an open neighbourhood $U$ of the origin 0 in $\mathbb{C}^{n}$ and denote by $\operatorname{Sing}(X)$ the set of singular points of $X$. Let $f:(X, 0) \rightarrow(\mathbb{C}, 0)$ be holomorphic and set $V=f^{-1}(0)$ and let $\operatorname{Sing}(V)$ the set of singular points of $V$. Let $B_{\epsilon_{0}}$ be a closed ball in $U$ of sufficiently small radius $\epsilon>0$, centred at $0 \in \mathbb{C}^{n}$ and let $S_{\epsilon}$ be its boundary.

As we mentioned in the introduction, there is a canonical pencil of real analytic hypersurfaces associated to $f$ (see also [S, RSV]) defined as follows. For each $\theta \in[0, \pi)$, let $\mathcal{L}_{\theta}$ be the line through 0 in $\mathbb{R}^{2}$ with an angle $\theta$ (with respect to the $x$-axis) and set $X_{\theta}=f^{-1}\left(\mathcal{L}_{\theta}\right)$. Then each $X_{\theta}$ is a real analytic hypersurface and $\left\{X_{\theta}\right\}$ is called the canonical pencil of $f$, its main properties are summarized in the following theorem.

Theorem 2.4 (Canonical Decomposition). Let $\left\{X_{\theta}\right\}$ be the canonical pencil of $f$. Then

i) The $X_{\theta}$ are all homeomorphic real analytic hypersurfaces of $X$ with singular set $\operatorname{Sing}(V) \cup\left(X_{\theta} \cap \operatorname{Sing}(X)\right)$. Their union is the whole space $X$ and they all meet at $V$, which divides each $X_{\theta}$ in two homeomorphic halves, i.e., $X_{\theta}=E_{\theta}^{+} \cup V \cup E_{\theta}^{-}$and $E_{\theta}^{+} \cong E_{\theta}^{-}$.

ii) If $\left\{S_{\alpha}\right\}$ is a Whitney stratification of $X$ adapted to $V$, i.e., $V$ is union of strata, then the intersection of the strata with each $X_{\theta}$ determines a Whitney stratification of $X_{\theta}$, and for each stratum $S_{\alpha}$ and each $X_{\theta}$, the intersection $S_{\alpha} \cap X_{\theta}$ meets transversally every sphere in $B_{\epsilon_{0}}$ centred at 0 . 
iii) There exist a small $\epsilon>0$ such that there is a uniform conical structure for all $X_{\theta}$, i.e., there is a homeomorphism

$$
h:\left(X \cap B_{\epsilon_{0}}, V \cap B_{\epsilon_{0}}\right) \rightarrow\left(\operatorname{Cone}\left(X \cap S_{\epsilon_{0}}\right) \text {, Cone }\left(V \cap S_{\epsilon_{0}}\right)\right)
$$

which restricted to each $X_{\theta}$ defines a homeomorphism

$$
\left(X_{\theta} \cap B_{\epsilon_{0}}\right) \cong \operatorname{Cone}\left(X_{\theta} \cap S_{\epsilon_{0}}\right) .
$$

The generalization of Theorems 2.1 and 2.3 is the following theorem.

Theorem 2.5 (Fibration Theorem). One has a commutative diagram of fibre bundles



where $\Psi(x)=(\operatorname{Re}(f(x)): \operatorname{Im}(f(x)))$ with fibre $\left(X_{\theta} \cap B_{\epsilon_{0}}\right) \backslash V, \Phi(x)=$ $\frac{f(x)}{|f(x)|}$ and $\pi$ is the natural two-fold covering. The restriction of $\Phi$ to the link $L_{X} \backslash L_{f}$ is the Milnor fibration $\phi$ in Theorem 2.3, while the restriction of $\Phi$ to the Milnor tube $f^{-1}\left(\partial D_{\eta}\right) \cap B_{\epsilon_{0}}$ is the Milnor-Lê fibration in Theorem 2.1 (up to multiplication by a constant), and the two fibrations are equivalent.

To prove Theorem 2.5 the authors introduce an auxiliary function called the spherefication of $f$, defined by

$$
\mathfrak{F}(x)=\|x\| \Phi(x)=\|x\| \frac{f(x)}{|f(x)|} .
$$

Notice that given $z \in \mathbb{C} \backslash\{0\}$ with $\theta=\arg z$, the fibre $\mathfrak{F}^{-1}(z)$ is the intersection of $E_{\theta}^{ \pm}$with the sphere $S_{|z|}$ of radius $|z|$ centred at 0 , and $\mathfrak{F}$ carries $S_{|z|} \backslash V$ into the circle around $0 \in \mathbb{R}^{2}$ of radius $|z|$.

Then they obtain the following fibration theorem.

Theorem 2.6. For $\epsilon_{0}>0$ sufficiently small, one has a fibre bundle

$$
\mathfrak{F}:\left(\left(X \cap B_{\epsilon_{0}}\right) \backslash V\right) \longrightarrow\left(D_{\epsilon_{0}} \backslash\{0\}\right),
$$

taking $x$ into $\|x\| \frac{f(x)}{|f(x)|}$, where $D_{\epsilon_{0}}$ is the disc in $\mathbb{R}^{2}$ centred at 0 with radius $\epsilon_{0}$. Furthermore, the restriction of $\mathfrak{F}$ to each sphere around 0 of radius $\epsilon \leq \epsilon_{0}$ is a fibre bundle over the corresponding circle of radius $\epsilon$, and this is the Milnor fibration $\phi$ in Theorem 2.3 up to multiplication by a constant. 
Idea of the proofs. Using Theorem 2.4-ii) it is proved that $\mathfrak{F}$ is a submersion on each strata in $X \backslash V$. This allows to construct on $\left(X \cap B_{\epsilon_{0}}\right) \backslash V$ two complete, stratified, vector fields: $\hat{v}$ is tangent to all the spheres in $B_{\epsilon}$ centred at 0 , and whose orbits are transverse to the $X_{\theta} \backslash V$ and permute them; $\hat{w}$ is transverse to all spheres in $B_{\epsilon_{0}}$ centred at 0 , and therefore, it is transverse to all fibres $\mathfrak{F}^{-1}(y)$. These vector fields give the local triviality of $\mathfrak{F}$ in Theorem 2.6. The vector field $\hat{v}$ also gives the local triviality of the restriction of $\mathfrak{F}$ to any sphere $S_{\epsilon}$ which is equivalent to the fibration $\phi$ since $\Phi=\frac{\mathfrak{F}(x)}{\|\widetilde{F}(x)\|}=\frac{f(x)}{\|f(x)\|}$. Also $\hat{v}$ gives the local triviality of $\Phi$ and $\Psi$ in Theorem 2.5.

Modifying $\hat{w}$ one gets a vector field $\tilde{w}$ which is transverse to all spheres in $B_{\epsilon_{0}}$ centred at 0 , it is also transverse to all the tubes $f^{-1}\left(\partial D_{\eta}\right)$ and it is tangent to the strata of each $X_{\theta} \backslash V$. This vector field gives the equivalence of the fibration on the tube and the one on the sphere.

The following corollary gives the relation between the Milnor fibers and the link of the $X_{\theta}$.

Corollary 2.7. Given $f:(X, 0) \rightarrow(\mathbb{C}, 0)$ and its Milnor fibration

$$
\phi=\frac{f}{|f|}: L_{X \epsilon} \backslash L_{f_{\epsilon}} \longrightarrow S^{1}
$$

one has that the union of every pair of fibres of $\phi$ over antipodal points of $S^{1}$ with the link $L_{f}$ is the link of a real analytic hypersurface $X_{\theta}$, which is homeomorphic to the link of $\{\operatorname{Re} f=0\}$. Moreover, if both $X$ and $f$ have an isolated singularity at 0 , then this homeomorphism is in fact a diffeomorphism and the link of each $X_{\theta}$ is diffeomorphic to the double of the Milnor fibre of $f$ regarded as a smooth manifold with boundary $L_{f}$.

Also a new Milnor-type fibration theorem is obtained in which it is not necessary to remove the zero locus of the function $f$.

Theorem 2.8. Let $\tilde{X}$ be the space obtained by the real blow-up of $V$, i.e., the blow-up of $(\operatorname{Re}(f), \operatorname{Im}(f))$. The projection $\tilde{\Psi}: \tilde{X} \rightarrow \mathbb{R P}^{1}$ is a topological fibre bundle with fibre $X_{\theta}$.

This implies that all the hypersurfaces $X_{\theta}$ are homeomorphic and can also used to prove Theorem 2.5.

Idea of the proof. The Whitney stratification of $X$ induces a canonical Whitney stratification on $\tilde{X}, \tilde{\Psi}$ is an stratified submersion and by Theorem 2.4-ii), the fibres of $\tilde{\Psi}$ are transverse to $\tilde{X} \cap\left(S_{\epsilon_{0}} \times \mathbb{R P}^{1}\right)$, the boundary of the compact set $\tilde{X} \cap\left(B_{\epsilon_{0}} \times \mathbb{R P}^{1}\right)$, so one can apply Thom-Mather First Isotopy Theorem to get the fibration. 


\section{Milnor FibRATIONS FOR REAL MAPS}

3.1. Fibrations for maps $f \bar{g}$ and meromorphic functions. It is natural to ask if, as in the holomorphic case, it is possible to have a fibration theorem for real analytic map germs $f:\left(\mathbb{R}^{n}, 0\right) \rightarrow\left(\mathbb{R}^{k}, 0\right)$ having an isolated critical value at $0 \in \mathbb{R}^{k}$, instead of having only $0 \in \mathbb{R}^{n}$ as an isolated critical point. This was first considered in [PS] where the authors generalized Theorem 2.1 for the case of real analytic maps $f: X \rightarrow \mathbb{R}^{k}$ on a real analytic variety $X$ of dimension $n>0$, with $0 \in \mathbb{R}^{k}$ as an isolated critical value and that satisfies Thom's $A_{f}$-condition and get the fibration corresponding to (2). Using this generalization they prove the following fibration theorem.

Let $X$ be an equidimensional, complex analytic variety in $\mathbb{C}^{N}$ of dimension $n$ with an isolated singularity at 0 . Let $f, g:(X, 0) \rightarrow(\mathbb{C}, 0)$ be germs of holomorphic functions such that $f^{-1}(0)$ and $g^{-1}(0)$ have no common irreducible components. Denote by $L_{X}$ the link of $X$ and by and $L_{f \bar{g}}$ is the link of $f \bar{g}$ in $X$.

Theorem 3.1. Suppose $f \bar{g}:(X, 0) \rightarrow(\mathbb{C}, 0)$ has an isolated critical value at $0 \in \mathbb{R}^{2} \cong \mathbb{C}$ and that it satisfies Thom's $A_{f}$-condition. Then one has a locally trivial fibration

$$
\Psi_{f \bar{g}}: L_{X} \backslash L_{f \bar{g}} \longrightarrow S_{\eta}^{1} .
$$

When $X$ has dimension 2 it is proved that for $f$ and $g$ with no common branch and $f \bar{g}$ with an isolated critical point at $0, f \bar{g}$ satisfies Thom's $A_{f}$-condition.

With the previous setting, also in [PS] the authors generalize a fibration theorem in $[\mathrm{BP}]$ for meromorphic functions $f / g$ which are semitame.

The meromorphic function $f / g$ takes values on $\mathbb{P}^{1}$. Let $V_{f g}=\{f g=$ 0 \} and denote by $L_{f g}$ the link of $f g$ in $X$.

Definition 3.2. Set $h=f / g$ and define the set

$$
M(f / g)=\left\{x \in X \backslash V_{f g} \mid T_{x}\left(\left(h^{-1}(h(x))\right) \subset T_{x} S_{\|x\|}^{2 N-1}\right\} .\right.
$$

The bifurcation set $B \subset \mathbb{P}^{1}$ of $f / g$ is the union of $\{0, \infty\}$ and the set of $c \in \mathbb{P}^{1}$ such that there exists a sequence $\left(x_{k}\right)_{k \in \mathbb{N}}$ in $M(f / g)$ such that

$$
\lim _{k \rightarrow \infty} x_{k}=0 \quad \text { and } \quad \lim _{k \rightarrow \infty}(f / g)\left(x_{k}\right)=c .
$$

The meromorphic function $f / g$ is semitame at 0 if $B=\{0, \infty\}$. 
Theorem $3.3([\mathrm{BP}])$. Let $f, g:(X, 0) \rightarrow(\mathbb{C}, 0)$ be holomorphic with no common branch. If $\mathrm{f} / \mathrm{g}$ is semitame at 0 , then the map

$$
\phi_{f / g}=\frac{f / g}{|f / g|}: \mathcal{L}_{X} \backslash L_{f g} \longrightarrow S^{1}
$$

is a locally trivial $C^{\infty}$ fibre bundle.

Notice that on $X \backslash V_{f g}$ one has $\frac{f \bar{g}}{|f \bar{g}|}=\frac{f / g}{|f / g|}$, and in fact one has that for $f, g:(X, 0) \rightarrow(\mathbb{C}, 0)$ be holomorphic with no common branch such that $f \bar{g}$ has an isolated critical value at 0 , it satisfies Thom's $A_{f \bar{g}^{-}}$ condition and $f / g$ is semitame at 0 the fibrations $\Psi_{f \bar{g}}$ and $\phi_{f / g}$ are topologically equivalent.

Recall that when $X$ has dimension $2, f \bar{g}$ satisfies Thom's $A_{f \bar{g}}$-condition, so in this case it is natural to compare the hypothesis: $f \bar{g}$ has an isolated critical value at 0 and $f / g$ is semitame at 0 , which gives fibrations $\Psi_{f \bar{g}}$ and $\phi_{f / g}$ respectively. In the case $X=\mathbb{C}^{2}$ these two hypothesis are equivalent.

Theorem 3.4. Let $f, g:\left(\mathbb{C}^{2}, 0\right) \rightarrow(\mathbb{C}, 0)$ be holomorphic germs such that $f^{-1}(0)$ and $g^{-1}(0)$ have no common factors. The following are equivalent :

(i) $f \bar{g}$ has an isolated critical value at 0 ,

(ii) The map $\Psi_{f \bar{g}}: L_{X} \backslash L_{f g} \rightarrow S^{1}$ is a fibration.

(iii) The map $\phi_{f / g}: L_{X} \backslash L_{f g} \rightarrow S^{1}$ is a fibration.

(iv) $\mathrm{f} / \mathrm{g}$ is semitame at 0 .

3.2. Fibrations of polar weighted homogeneous polynomials. In [RSV] twisted Brieskorn-Pham polynomials were defined (see Example 3.8), which were the first examples of polar weighted homogeneous polynomials. Inspired by these examples, in [Ci, O] Polar weighted homogeneous polynomials were defined, they are real analytic maps which generalize complex weighted homogeneous polynomials. This family of polynomials have $0 \in \mathbb{C}$ as its only critical value. Hence they are examples of real analytic maps which does not necessarily have isolated critical point and have Milnor Fibrations on the Milnor tube and on the sphere, and the two fibrations are equivalent.

Consider $\mathbb{C}^{n}$ with coordinates $z_{1}, \ldots, z_{n}$. As usual, let $\bar{z}_{j}$ be the complex conjugate of $z_{j}$. Writing $z_{j}=x_{j}+i y_{j}$, we have that considering $\mathbb{C}^{n}$ with coordinates $z_{1}, \ldots, z_{n}, \bar{z}_{1}, \ldots, \bar{z}_{n}$ is equivalent to consider it as a $2 n$-dimensional real vector space with coordinates $x_{1}, y_{1}, \ldots, x_{n}, y_{n}$. To simplify notation we shall write $\mathbf{z}=\left(z_{1}, \ldots, z_{n}\right), \overline{\mathbf{z}}=\left(\bar{z}_{1}, \ldots, \bar{z}_{n}\right)$. Also set $\mathbb{C}^{*}=\mathbb{C}-\{0\}$. 
Definition 3.5. Let $p_{j}, u_{j}$ with $j=1, \ldots, n$, positive integers such that

$$
\operatorname{gcd}\left(p_{1}, \ldots, p_{n}\right)=1, \quad \operatorname{gcd}\left(u_{1}, \ldots, u_{n}\right)=1 .
$$

Let $\tau \in \mathbb{C}^{*}$ written in its polar form $\tau=t \lambda$, with $t \in \mathbb{R}^{+}$and $\lambda \in S^{1}$, that is, $t=|\tau|$ and $\lambda=e^{i \arg \tau}$.

A polar $\mathbb{C}^{*}$-action on $\mathbb{C}^{n}$ with radial weights $\left(p_{1}, \ldots, p_{n}\right)$ and polar weights $\left(u_{1}, \ldots, u_{n}\right)$ is given by

$$
t \lambda \cdot(\mathbf{z})=\left(t^{p_{1}} \lambda^{u_{1}} z_{1}, \ldots, t^{p_{n}} \lambda^{u_{n}} z_{n}\right) .
$$

In fact, a polar $\mathbb{C}^{*}$-action is the combination of two actions: a $\mathbb{R}^{+}$-action given by the weights $\left(p_{1}, \ldots, p_{n}\right)$, and a $S^{1}$-action given by the weights $\left(u_{1}, \ldots, u_{n}\right)$.

Definition 3.6. Let $f: \mathbb{C}^{n} \rightarrow \mathbb{C}$ be a polynomial in the $2 n$ variables $z_{1}, \ldots, z_{n}, \bar{z}_{1}, \ldots, \bar{z}_{n}$. Hence, we can see $f$ as a real analytic function.

Let $a$ and $c$ be positive integers. We say that $f$ is polar weighted homogeneous with radial weight type $\left(p_{1}, \ldots, p_{n} ; a\right)$ and polar weight type $\left(u_{1}, \ldots, u_{n} ; c\right)$ if the following functional identity holds

$$
f(t \lambda \cdot(\mathbf{z}))=t^{a} \lambda^{c} f(\mathbf{z}), \quad t \in \mathbb{R}^{+}, \lambda \in S^{1},
$$

where $t \lambda \cdot(\mathbf{z})$ is a polar $\mathbb{C}^{*}$-action. In other words, it is weighted homogeneous of degree $a$ with respect to the $\mathbb{R}^{+}$-action with weights $\left(p_{1}, \ldots, p_{n}\right)$ and it is weighted homogeneous of degree $c$ with respect to the $S^{1}$-action with weights $\left(u_{1}, \ldots, u_{n}\right)$.

Example 3.7. Weighted homogeneous polynomials are a particular case of polar weighted homogeneous polynomials with no $\bar{z}_{j}$ for $j=$ $1, \ldots, n$ and with $p_{j}=u_{j}$ and $a=c$. In particular, Pham-Brieskorn polynomials (1).

Example 3.8. A polynomial in $\mathbb{C}^{n}$ of the form

$$
\nu_{1} z_{1}^{a_{1}} \bar{z}_{\sigma(1)}+\cdots+\nu_{n} z_{n}^{a_{n}} \bar{z}_{\sigma(n)}
$$

was called in [RSV] a twisted Brieskorn-Pham polynomial of class $\left\{a_{1}, \ldots, a_{n} ; \sigma\right\}$, where each $a_{j} \geq 2, j=1, \ldots, n$, the $\nu_{j}$ are non-zero complex numbers and $\sigma$ is a permutation of the set $\{1, \ldots, n\}$ called the twisting.

In [RSV] it is proved that twisted Brieskorn-Pham polynomials are polar weighted homogeneous. It is also proved that they have isolated critical point and that they satisfy the strong Milnor condition.

Remark 3.9. The sum of two polar weighted homogeneous polynomials is again a polar weighted homogeneous polynomial. 
Using a generalization of Euler identities for weighted homogeneous polynomials it is proved that $0 \in \mathbb{C}$ is the only critical value of polar weighted homogeneous polynomials. Then as for complex weighted homogeneous polynomials one has the following fibration theorem.

Let $V=f^{-1}(0)$, let $S_{\epsilon}^{2 n-1} \subset \mathbb{C}^{n}$ be a sphere of radius $\epsilon$ around 0 and let $K_{\epsilon}=S_{\epsilon}^{2 n-1} \cap V$.

Proposition 3.10. The restriction $f:\left(\mathbb{C}^{n}-V\right) \rightarrow \mathbb{C}^{*}$ is a locally trivial fibration. Its monodromy is given by the map

$$
h(\mathbf{z})=e^{2 \pi i / c} \cdot \mathbf{z} .
$$

Moreover, the map

$$
\phi=\frac{f}{|f|}:\left(S_{\epsilon}^{2 n-1} \backslash K_{\epsilon}\right) \rightarrow S^{1},
$$

is a locally trivial fibration for any $\epsilon>0$.

Let $f_{\mid}: f^{-1}\left(S^{1}\right) \rightarrow S^{1}$ be the restriction of the fibration $f:\left(\mathbb{C}^{n}-\right.$ $V) \rightarrow \mathbb{C}^{*}$ of Proposition 3.10 to $S^{1}$.

Proposition 3.11. The fibration $f_{\mid}: f^{-1}\left(S^{1}\right) \rightarrow S^{1}$ is equivalent to the Milnor fibration $\phi: S_{\epsilon}^{2 n-1} \backslash K_{\epsilon} \rightarrow S^{1}$.

In [Ci] it is also proved a Join Theorem, which says that the Milnor fiber of the sum of two polar weighted homogeneous polynomials in different variables, is homotopically equivalent to the join of the Milnor fibers of the summands.

Polar weighted homogeneous polynomials are mixed analytic functions which is a wider class of real analytic functions recently defined in [O1]. There, the author gives a condition to guarantee the existence of Milnor Fibrations for mixed analytic functions.

3.3. Milnor conditions (a) and (b) and E-analytic maps. An interesting improvement toward the existence of Milnor fibrations for real analytic map germs $f:\left(\mathbb{R}^{n}, 0\right) \rightarrow\left(\mathbb{R}^{k}, 0\right), m \geq k \geq 2$, was given in [Ma]. The author defined two conditions under which is possible to guarantee the existence of Milnor fibrations for non-isolated singularities, more precisely, for map $f$ with isolated critical value at $0 \in \mathbb{R}^{k}$.

In order to describe this result and following the author's notation in [Ma], let $f(x)=\left(f_{1}(x), \ldots, f_{k}(x)\right)$ be a representant of the germ defined in a small neighborhood of origin $U, \nabla f_{i}(x)$ the gradient map of the coordinates functions $f_{i}$, for $i=1, \ldots, k$. Denote by $\mathfrak{U}=\sum(f)$ the singular locus of $f$ and $\rho(x)=\|x\|^{2}$ the square of distance function from the origin. Define

$\mathfrak{B}:=\left\{x \in U \mid \nabla f_{i}(x), \ldots, \nabla f_{k}(x), \nabla \rho(x)\right.$, are linearly dependent $\}$. 
Definition 3.12. We say that a map germ $f$ satisfies Milnor condition (a) at the origin 0 if, and only if, $0 \notin \overline{\mathfrak{U} \backslash V(f)}$.

Definition 3.13. We say that a map germ $f$ satisfies Milnor condition (b) at the origin 0 if, and only if, 0 is an isolated point of (or, is not in) $V(f) \cap \overline{\mathfrak{B} \backslash V(f)}$.

It is easy to see that Milnor condition (a) means that in a small neighborhood of origin $\sum(f) \subset V(f)$, i.e, $0 \in \mathbb{R}^{k}$ is an isolated critical value. Milnor condition (b) implies that there exists a small enough $\epsilon_{0}>0$, such that for each $0<\epsilon \leq \epsilon_{0}$, there exists $\eta, 0<\eta \ll \epsilon$, such that $f^{-1}\left(B_{\eta}^{k}-\{0\}\right) \cap S_{\epsilon}^{n-1} \cap \mathfrak{B}=\varnothing$, where $B_{\eta}^{k}-\{0\}$ stand for the punctured closed ball in $\mathbb{R}^{k}$ of radius $\eta$.

Definition 3.14. If $f$ satisfies Milnor conditions (a) and (b) at the origin, we say that $\epsilon$ is a Milnor radius for $f$ at the origin, if both conditions holds into a closed ball $B_{\epsilon} \subset \mathbb{R}^{n}$.

Theorem 3.15 ([Ma]). Suppose that a real analytic map $f$ has a Milnor radius $\epsilon_{0}>0$. Then, for all $\epsilon, 0<\epsilon \leq \epsilon_{0}$, there exists $\eta, 0<\eta \ll \epsilon_{0}$, such that

$$
f_{\mid}: B_{\epsilon} \cap f^{-1}\left(B_{\eta}^{k}-\{0\}\right) \rightarrow B_{\eta}^{k}-\{0\}
$$

is the projection of a smooth locally trivial fibration.

Idea of proof. Since $f$ has a Milnor radius $\epsilon_{0}>0$, we have that for all $0<\epsilon \leq \epsilon_{0}, \sum(f) \cap B_{\epsilon} \subset V(f) \cap B_{\epsilon}$. It means that, $0 \in \mathbb{R}^{k}$ is an isolated critical value of $f$, i.e, $f_{\mid}: B_{\epsilon}^{\circ} \backslash V(f) \rightarrow R^{k}$ is a smooth submersion, where $B^{\circ}$ stands for an open ball. It follows from Milnor condition (b), and the remark above that, for each $\epsilon$ there exists $\eta, 0<\eta \ll \epsilon_{0}$, such that

$$
f_{\mid}: S_{\epsilon}^{n-1} \cap f^{-1}\left(B_{\eta}^{\circ}-\{0\}\right) \rightarrow B_{\eta}^{\circ}-\{0\}
$$

is a submersion on the boundary of the closed ball $B_{\epsilon}$. Now, combining these two conditions, we get that for each $\epsilon$ we can choose $\eta$ such that

$$
f_{\mid}: B_{\epsilon} \cap f^{-1}\left(B_{\eta}^{\circ}-\{0\}\right) \rightarrow B_{\eta}^{\circ}-\{0\}
$$

is a smooth, proper submersion and by Ehresmann Fibration Theorem it is a locally trivial fibration.

Even in $[\mathrm{Ma}]$ the author gave the following analytic condition to guarantee the existence of Milnor's radius of a map $f$.

Definition 3.16. We say that a real analytic map germ $f:\left(\mathbb{R}^{n}, 0\right) \rightarrow$ $\left(\mathbb{R}^{k}, 0\right), m \geq k \geq 2$, satisfies the strong Lojasiewicz inequality at the origin or is $\mathbf{E}$-analytic at the origin, if, and only if, there exists 
an open neighborhood $U \ni 0$, and constants $c>0,0<\theta<1$, such that, for all $x \in U$, the following holds:

$$
|f(x)|^{\theta} \leq c . \min _{\left|\left(a_{1}, \ldots, a_{k}\right)\right|=1}\left\{\left|a_{1} \cdot \nabla f_{1}(x)+\cdots+a_{k} \cdot \nabla f_{k}(x)\right|\right\} .
$$

It is easy to see that, E-analytic maps always satisfies Milnor condition (a). In [Ma], using a technique started in [HL], the author proved the following result:

Theorem 3.17 ([Ma]). If a real analytic map pair $f=(P, Q)$ : $\left(\mathbb{R}^{n}, 0\right) \rightarrow\left(\mathbb{R}^{2}, 0\right)$ is E-analytic at the origin, then Milnor condition (b) holds. i.e, there exist a Milnor radius for the map $f$. Therefore, (applying Theorem.3.15) the local Milnor fibration exists.

In general, i.e, for real analytic map germ $f:\left(\mathbb{R}^{n}, 0\right) \rightarrow\left(\mathbb{R}^{k}, 0\right), m \geq$ $k>2$, the author posed the following open questions:

Question 2. Do general Ł-analytic maps satisfy Milnor condition (b)?

Question 3. Let $f:\left(\mathbb{R}^{n}, 0\right) \rightarrow\left(\mathbb{R}^{2}, 0\right)$ be an E-analytic map that also satisfies the strong Milnor condition. Are the two fibrations equivalent?

3.4. Real Milnor fibration and open book decompositions. As we have mentioned before, we cannot expect in general that given a real analytic map germ $f:\left(\mathbb{R}^{n}, 0\right) \rightarrow\left(\mathbb{R}^{k}, 0\right)$, with isolated critical point at the origin, the projection $\frac{f}{\|f\|}: S_{\epsilon}^{m-1} \backslash K_{\epsilon} \rightarrow S^{k-1}$ will be the projection map of a smooth locally trivial fibration, i.e, for $k=2$, that $f$ will satisfy the strong Milnor condition at the origin.

In [ST] the second author and M. Tibar used the idea of open book decomposition in higher dimensions to get a characterization of this kind of fibrations, for all dimensions $k \geq 2$, on the class of $\mathcal{K}$-finite map germs. Their main result is:

Theorem 3.18 ([ST]). Let $f:\left(\mathbb{R}^{n}, 0\right) \rightarrow\left(\mathbb{R}^{k}, 0\right)$ be a real analytic map germ and suppose that for all small enough radii $\epsilon>0, \sum(f) \cap$ $V(f) \cap B_{\epsilon} \subseteq\{0\}$. Then, the map $\frac{f}{\|f\|}: S_{\epsilon}^{n-1} \backslash K_{\epsilon} \rightarrow S^{k-1}$ is the map projection of a locally trivial fibration if, and only if, it is a submersion for all $\epsilon$. Moreover, if the map is weighed-homogeneous with isolated singularity at origin, this fibration is smooth and fiber-equivalent to the Milnor fibration given in Theorem.1.3. 
Idea of proof. Let $f(x)=\left(f_{1}(x), \ldots, f_{k}(x)\right)$ and $s=\left(s_{1}, \ldots, s_{k}\right) \in$ $\mathbb{R}^{k} \backslash\{0\}$, now consider $[s]=\left(s_{1}: \ldots: s_{k}\right) \in \mathbb{P}^{k-1}(\mathbb{R})$ and define the analytic set

$$
X:=\left\{x \in B_{\epsilon_{0}} \times \mathbb{P}^{k-1}(\mathbb{R}): \operatorname{rank}\left[\begin{array}{c}
f_{1}(x) \cdots f_{k}(x) \\
s_{1} \cdots s_{k}
\end{array}\right]<2\right\} .
$$

Define $\pi: X \rightarrow \mathbb{P}^{k-1}(\mathbb{R})$ and $p: X \rightarrow B_{\epsilon_{0}}$ and denote $X_{[s]}$ the fiber of $\pi$ over $[s] \in \mathbb{P}^{k-1}(\mathbb{R})$. It is easy to see that $V \times \mathbb{P}^{k-1}(\mathbb{R}) \subset X$ and $V \times[s] \subset X_{[s]}$, for all $[s]$. Also, denote

$$
X_{[s]}^{+}=\left(\frac{f}{\|f\|}\right)^{-1}\left(\frac{s}{\|s\|}\right) \text { and } X_{[s]}^{-}=\left(\frac{f}{\|f\|}\right)^{-1}\left(-\frac{s}{\|s\|}\right) \text {. }
$$

The three conditions below holds:

1) $X_{[s]} \backslash V$ is a disjoint union of $X_{[s]}^{+}$and $X_{[s]}^{-}$;

2) The first factor projection $p: X \rightarrow B_{\epsilon_{0}}$ is actually a blow up along $V$, i.e, $p: X \backslash\left(V \times \mathbb{P}^{k-1}(\mathbb{R})\right) \rightarrow B_{\epsilon_{0}} \backslash V$ is an analytic isomorphism;

3) The submersion condition on hypothesis means that: for all $0<\epsilon \leq$ $\epsilon_{0}, X_{[s]} \backslash V$ is an analytic manifold for all $[s]$ and it is transverse to all small sphere $S_{\epsilon}$.

Now the equivalence follows similarly to the construction given in [Mi1] and [RS].

We observe that, if $f$ has isolated singular point at origin, i.e. $\sum(f)=$ $\{0\}$, our main hypothesis is fulfilled, so the following result is an immediate consequence:

Corollary 3.19. A necessary and sufficient condition for a pair of isolated singular map germ $f=(P, Q):\left(\mathbb{R}^{n}, 0\right) \rightarrow\left(\mathbb{R}^{2}, 0\right)$ satisfies the strong Milnor condition at the origin, is that the projection map $\frac{f}{\|f\|}: S_{\epsilon}^{m-1} \backslash K_{\epsilon} \rightarrow S^{1}$ be a submersion, for all $\epsilon>0$ small enough.

3.5. Real Milnor fibrations and $d$-regularity. Using the ideas and constructions given in [CSS] for holomorphic functions, in [CSS] for the case $k=2$ and [CSS1] for the general case, the authors introduce a condition called $d$-regularity, which is a necessary and sufficient condition to have several kinds of fibrations associated to a real analytic map $f:\left(\mathbb{R}^{n}, 0\right) \rightarrow\left(\mathbb{R}^{k}, 0\right)$ with an isolated critical value at $0 \in \mathbb{R}^{k}$.

Let $f:(U, 0) \rightarrow\left(\mathbb{R}^{k}, 0\right)$ be a non-constant real analytic map defined on an open neighbourhood of the origin $0 \in \mathbb{R}^{n}$, with a critical point at 0 and which is a submersion at each $x \notin V=f^{-1}(0)$, i.e., $0 \in \mathbb{R}^{k}$ is an isolated critical value. As in $\S 2.2$ we define the canonical pencil of $f$, which is a family of real analytic spaces, parametrised by $\mathbb{R P}^{k-1}$, as 
follows: For each $\ell \in \mathbb{R} \mathbb{P}^{k-1}$, consider the line $\mathcal{L}_{\ell} \subset \mathbb{R}^{k}$ passing through the origin corresponding to $\ell$ and set

$$
X_{\ell}=\left\{x \in U \mid f(x) \in \mathcal{L}_{\ell}\right\}
$$

Then each $X_{\ell}$ is a real analytic variety. It is easy to see that these varieties meet at $V$ and away from it they are smooth submanifolds of $U$ of dimension $n-k+1$.

Definition 3.20. The map $f$ is said to be $d$-regular at 0 if there exists a metric $\rho$ induced by some positive definite quadratic form and there exists $\epsilon_{0}>0$ such that every sphere (for the metric $\rho$ ) of radius $\leq \epsilon_{0}$ centred at 0 meets every $X_{\ell} \backslash V$ transversely whenever the intersection is not empty. We shall also say that $f$ is $d$-regular with respect to the metric $\rho$.

Example 3.21. The first three examples are $d$-regular at 0 for the usual metric:

1) By $\left[\right.$ Mi1, Lem. 5.9], every holomorphic germ $f:\left(\mathbb{C}^{n}, 0\right) \rightarrow(\mathbb{C}, 0)$ is $d$-regular (see $[\mathrm{CSS}]$ ).

2) By $[\mathrm{PS}]$, given $f, g:\left(\mathbb{C}^{2}, 0\right) \rightarrow(\mathbb{C}, 0)$ holomorphic germs (see $\left.\S 3.1\right)$ :

- If $f \bar{g}$ has an isolated critical value at $0 \in \mathbb{C}$, then it is $d$-regular.

- If $f / g:\left(\mathbb{C}^{n}, 0\right) \rightarrow(\mathbb{C}, 0)$ is semitame at 0 , then it is $d$-regular.

3) By $[\mathrm{Ci}]$ (see $\S 3.2$ ), Polar weighted homogeneous polynomials are $d$ regular.

4) Assume $f:\left(\mathbb{R}^{n}, 0\right) \rightarrow\left(\mathbb{R}^{p}, 0\right)$ is real weighted homogeneous with isolated critical value at 0 . The orbits of the action of $\mathbb{R}^{*}$ are obviously tangent to each $X_{\ell}$ and transversal to all spheres centered at 0 . Hence $f$ is $d$-regular.

5) By $[\mathrm{RS}]$, every map-germ $g:\left(\mathbb{R}^{n+2}, 0\right) \rightarrow\left(\mathbb{R}^{2}, 0\right)$ for which its pencil is $c$-regular (in the sense of $\mathrm{K}$. Bekka) with respect to the control function defined by a metric $d$ in $\mathbb{R}^{n+2}$, is $d$-regular.

The condition of $d$-regularity is equivalent to the condition given in $\S 3.4$ that the map

$$
\phi=\frac{f}{\|f\|}: S_{\epsilon}^{n-1} \backslash K_{\epsilon} \rightarrow S^{k-1}
$$

is a submersion for any sufficiently small $\epsilon$. In fact, one has the following characterizations of $d$-regularity in terms of the spherification of $f$.

As in $\S 2.2$, define the map $\Phi: U \backslash V \rightarrow S^{k-1}$ by $\Phi(x)=\frac{f(x)}{\|f(x)\|}$ and the spherefication $\mathfrak{F}: U \backslash V \rightarrow \mathbb{R}^{k} \backslash\{0\}$ of $f$ by $\mathfrak{F}(x)=\|x\| \Phi(x)$.

Proposition 3.22. Let $B_{\epsilon_{0}} \subset U$ be a ball centered at $0 \in \mathbb{R}^{n}$. Let $f:\left(B_{\epsilon_{0}}, 0\right) \rightarrow\left(\mathbb{R}^{k}, 0\right)$. Set $V=f^{-1}(0)$ and $K_{\epsilon}=V \cap S_{\epsilon}$. The following are equivalent: 
(1) The map $f$ is d-regular at 0.

(2) For each sphere $S_{\epsilon}$ in $\mathbb{R}^{n}$ centred at 0 of radius $\epsilon \leq \epsilon_{0}$, the restriction map $\mathfrak{F}_{\epsilon}: S_{\epsilon} \backslash V \rightarrow S_{\epsilon}^{k-1}$ of $\mathfrak{F}$ is a submersion.

(3) The spherefication map $\mathfrak{F}$ is a submersion at each $x \in B_{\epsilon_{0}} \backslash V$.

(4) The map $\phi=\frac{f}{\|f\|}: S_{\epsilon} \backslash K_{\epsilon} \longrightarrow S^{k-1}$ is a submersion for every sphere $S_{\epsilon}$ with $\epsilon \leq \epsilon_{0}$.

Idea of proof. The equivalences are straightforward from the definitions of $d$-regularity and the spherefication map. The important point is to notice that $\Phi(x)=\frac{f(x)}{\|f(x)\|}=\frac{\mathfrak{F}(x)}{\|\mathfrak{F}(x)\|}$.

The main result of [CSS1] is the following real analogue of Theorems 2.5 and 2.6 .

Theorem 3.23 (Fibration Theorem). Fix a metric $\rho$ in $\mathbb{R}^{n}$ and let $B_{\epsilon_{0}}$ be a sufficiently small open ball around $0 \in \mathbb{R}^{n}$ with respect to this metric. Let $f:=\left(f_{1}, \ldots, f_{k}\right): \mathbb{R}^{n} \rightarrow \mathbb{R}^{k}$ be a real analytic mapgerm, which is a submersion away from $V=f^{-1}(0)$. Define the map $\Phi: B_{\epsilon_{0}} \backslash V \rightarrow S^{k-1}$ by $\Phi=\frac{f}{\|f\|}$ and the spherefication map of $f$ by

$$
\mathfrak{F}: B_{\epsilon_{0}} \backslash V \rightarrow D_{\epsilon_{0}} \backslash\{0\}, \quad \mathfrak{F}(x)=\|x\| \Phi(x),
$$

where $D_{\epsilon_{0}}$ is a ball in $\mathbb{R}^{k}$ centred at 0 with radius $\epsilon$. The following are equivalent:

1. The map $f$ is d-regular at 0 .

2. The map $\mathfrak{F}: B_{\epsilon_{0}} \backslash V \rightarrow \mathfrak{F}\left(B_{\epsilon_{0}} \backslash V\right) \subseteq D_{\epsilon_{0}} \backslash\{0\}$ is a differentiable fibre bundle. Furthermore, for every sphere $S_{\epsilon}$ centred at 0 of radius $\epsilon<\epsilon_{0}$, the restriction $\mathfrak{F}_{\epsilon}: S_{\epsilon} \backslash V \rightarrow \mathfrak{F}_{\epsilon}\left(S_{\epsilon} \backslash V\right) \subseteq S_{\epsilon}^{k-1}$ of $\mathfrak{F}$ is also a differentiable fibre bundle and this is the map $\phi$ in (7) up to multiplication by a constant.

3. One has a commutative diagram of smooth fibre bundles

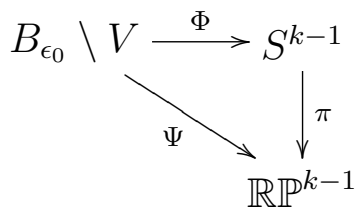

where $\Psi(x)=\left(f_{1}(x): \cdots: f_{k}(x)\right)$ has fibre $\left(X_{\ell} \cap B_{\epsilon_{0}}\right) \backslash V$ and $\pi$ is the natural two-fold covering.

4. Let $K_{\epsilon}=V \cap S_{\epsilon}$. Restricting to $S_{\epsilon} \backslash K_{\epsilon}$ one has a corresponding diagram of smooth fibre bundles on $S_{\epsilon} \backslash K_{\epsilon}$ for every sphere $S_{\epsilon}$ centred 
at 0 of radius $\epsilon<\epsilon_{0}$ :

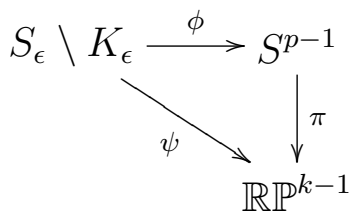

where $\psi=\left.\Psi\right|_{S_{\epsilon} \backslash K_{\epsilon}}$ and $\phi=\frac{f}{\|f\|}: S_{\epsilon} \backslash K_{\epsilon} \rightarrow S^{k-1}$.

Remark 3.24. In Theorem 3.23 the maps $\mathfrak{F}, \Psi, \Phi, \psi$ and $\phi$ are not necessarily surjective, so they are fibrations over their respective images.

Idea of the proof. The proof is analogue to the proofs of Theorems 2.5 and 2.6. By Proposition 3.22 the spherefication map $\mathfrak{F}$ is a submersion. This allows to prove the local triviallity of the fibrations mentioned in the Theorem using the fact that $\Phi(x)=\frac{f(x)}{\|f(x)\|}=\frac{\mathfrak{F}(x)}{\|\mathfrak{F}(x)\|}$ (see [CSS1] for details).

If $f$ satisfies Thom's $A_{f}$-condition, one has the fibration on the Milnor tube, and if $f$ is also $d$-regular this fibration is equivalent to the fibration on the sphere:

Theorem 3.25. Let $f:(U, 0) \rightarrow\left(\mathbb{R}^{k}, 0\right)$ be real analytic, with an isolated critical value at $0 \in \mathbb{R}^{k}$. Then:

a) If $V:=f^{-1}(0)$ has dimension more than 0 and $f$ has the Thom property at 0 , then one has a locally trivial fibration

$$
f: B_{\epsilon_{0}} \cap f^{-1}\left(\partial D_{\delta}\right) \longrightarrow \partial D_{\delta}
$$

where $D_{\delta} \subset \mathbb{R}^{k}$ is the disc of radius $\delta$ around $0 \in \mathbb{R}^{k}, \epsilon>>\delta>0$.

$b)$ If $f$ has the Thom property at $0, V$ has dimension more than 0 and $f$ is d-regular at 0 , then the two fibrations above, one on the Milnor tube (8), the other on the sphere (7), are equivalent.

Idea of the proof. Item a) follows from the generalization of Theorem 2.1 given in [PS] (see §3.1). Item b) is proved constructing a vector field as in the proof of Theorem 2.5. (see Idea of proofs in page 9).

\section{ACKnowledgements}

The idea of this paper started when the second author was developing his post-doc in the Northeastern University/Boston, under supervision of Prof. T. Gaffney/NEU. He would like to thank the Brazilian agency CNPq, grant PDE, number: 200643/2007-0 for supporting his stay and make it possible and also to all NEU staff for making his visit pleasant and productive. Thanks !

The first author thanks the second author for inviting him to write the present article. He was partially supported by CONACYT J-49048-F, Mexico. 


\section{REFERENCES}

[A] Norbert A'Campo. Le nombre de Lefschetz d'une monodromie. Nederl. Akad. Wetensch. Proc. Ser. A $\mathbf{7 6}=$ Indag. Math., 35:113-118, 1973.

[BP] A. Bodin and A. Pichon, Meromorphic functions, bifurcation sets and fibred links, Math. Res. Lett. 14(3) (2007) 413-422.

[CL] P. T. Church and K. Lamotke, Non-trivial polynomial isolated singularities, Nederl. Akad. Wetensch. Proc. Ser. A 78=Indag. Math. 37 (1975) 149-154.

[Ci] J. L. Cisneros-Molina, Join theorem for polar weighted homogeneous singularities, in Singularities II: Geometric and Topological aspects, Proc. of the Conference in honour of Lê Dũng Tráng, eds. J. P. Brasselet et al., (Cuernavaca, Mexico, 2007), Contemporary Mathematics, 475 (2008) 43-59.

[CSS] J. L. Cisneros-Molina, J. Snoussi, J. Seade, Refinements of Milnor's fibration theorem, preprint (2007), arXiv:0712.244.

[CSS1] J. L. Cisneros-Molina, J. Snoussi, J. Seade, Milnor Fibrations and $d$ regularity for real analytic Singularities, preprint, 2008.

[Du] Alan H. Durfee. Neighborhoods of algebraic sets. Trans. Amer. Math. Soc., 276(2):517-530, 1983.

[Ga] Terence T. Gaffney. Non isolated completed intersection singularities and the $A_{f}$-condition. Singularities I: Algebraic and Analytic aspects, Proc. of the Conference in honour of Lê Dũng Tráng, eds. J. P. Brasselet et al., (Cuernavaca, Mexico, 2007), Contemporary Mathematics, 474 (2008), 85-94.

[H] H. Hamm, Lokale topologische Eigenschaften komplexer Räume, Math Ann. 191, 235-252, 1971.

[HL] H. Hamm and L. Drung Trang Um Théorème de Zariski du type de Lefschetz. Ann. Sci. Éc. Norm. Sup.,(6), serie 4, 317-366, 1973.

[Hi] H. Hironaka. Stratification and flatness. In P. Holm, editor, Real and complex singularities (Proc. Ninth Nordic Summer School/NAVF Sympos. Math., Oslo, August 5-25, 1976), pages 199-265. Sijthoff and Noordhoff, Alphen aan den Rijn, 1977.

[Ja] A. Jaquemard, Fibrations de Milnor pour des applications réelles, Boll. Un. Mat. Ital., vol.37, 1, pp.45-62, 3-B,1989.

[Ja1] A. Jacquemard, Thèse 3ème cycle Université de Dijon, 1982.

[Le] Dũng Tráng Lê. Vanishing cycles on complex analytic sets. Sûrikaisekikenkyûsho Kókyûroku, (266):299-318, 1976. Various problems in algebraic analysis (Proc. Sympos., Res. Inst. Math. Sci., Kyoto Univ., Kyoto, 1975).

[Le1] Dũng Tráng Lê. Some remarks on relative monodromy. In P. Holm, editor, Real and complex singularities (Proc. Ninth Nordic Summer School/NAVF Sympos. Math., Oslo, August 5-25, 1976), pages 397-403. Sijthoff and Noordhoff, Alphen aan den Rijn, 1977.

[Lo] E. Looijenga, A note on polynomial isolated singularities, Indag. Math. 33 (1971) 418-421.

[Lo1] E. Looijenga, Isolated Singular Points on Complete Intersections, London Math. Soc. Lect. Notes. Ser. 77, 1984.

[Ma] D. Massey, Real Analytic Milnor Fibrations and a Strong Lojasiewicz Inequality, available on arXiv:math/0703613, 2008.

[Mat] John Mather. Notes on Topological Stability. Harvard University, July 1970. 
[Mi] John Milnor. On isolated singularities of hypersurfaces. Preprint June 1966. Unpublished.

[Mi1] J.Milnor, Singular points of complex hypersurfaces, Ann. of Math. Studies 61, Princeton University Press, 1968.

[O] Mutsuo Oka. Topology of Polar Weighted Homogeneous polynomials. Preprint, arXiv:0801.3708v1 [math.AG], to appear in Kodai Math. J., January 2008.

[O1] Mutsuo Oka. Non-degenerated mixed functions. Preprint, 2008.

[PS] A. Pichon and J. Seade, Fibered multilinks and singularities $f \bar{g}$, to appear in Math. Annalen.

[RS] M.A.S.Ruas and R. Santos, Real Milnor Fibrations and $(C)$-regularity, Manuscripta Math., 117, (2005), no. 2, 207-218.

[RSV] M.A.S.Ruas, J.Seade and A.Verjovsky On Real Singularities with a Milnor Fibration, Trends in Singularities, Birkhauser, 2002.

[Sa] R. dos Santos, Uniform (m)-condition and strong Milnor fibrations, in Singularities II: Geometric and Topological aspects, Proc. of the Conference in honour of Lê Dũng Tráng, eds. J. P. Brasselet et al., (Cuernavaca, Mexico, 2007), Contemporary Mathematics, 475 (2008) 43-59.

[ST] R.N. Araújo dos Santos and M. Tibar, Real map germs and higher open books, available on arXiv: 0801.3328, 2008.

[S] J.Seade, Open Book Decompositions Associated to Holomorphic Vector Fields, Bol.Soc.Mat.Mexicana(3), vol.3 , pp.323-336, 1997.

[S2] J.Seade, On the topology of isolated singularities in analytic spaces. Progress in Mathematics, 241. Birkhäuser Verlag, Basel, 2006.

[S3] José Seade. On Milnor's fibration theorem for real and complex singularities. In Singularities in geometry and topology, pages 127-158. World Sci. Publ., Hackensack, NJ, 2007.

Instituto de Matemáticas, Unidad Cuernavaca, Universidad Nacional Autónoma de México, Av. Universidad s/n, Lomas de Chamilpa, 62210 Cuernavaca, Morelos, A. P. 273-3, México

E-mail address: jlcm@matcuer.unam.mx

Instituto de Ciências Matemáticas e de Computação, Universidade de São Paulo, Av. Trabalhador São-Carlense, 400 - Centro Postal Box 668, São Carlos - SÃo Paulo - Brazil

Postal Code 13560-970, SÃo Carlos, SP, Brazil.

E-mail address: rnonato@icmc.usp.br 